\title{
Smart Sensor Design Analysis in Brain Machine Interface using Labview
}

\author{
Mamatha M.N \\ Research Scholar, Vinayaka \\ Mission University, Salem.
}

\author{
Dr S. Ramachandran \\ S. J. B. Institute of \\ Technology, Bangalore.
}

\author{
Dr M.Chandrasekaran \\ Government college of \\ Engineering, Salem.
}

\begin{abstract}
This paper describes a sensory system for implementing a human-computer interface based on bio-potential signal acquisition. The designed acquisition system captures electro oculogram, EEG and transmits them via the RF protocol to a nearby robot. The human machine interface developed will assist a partially paralyzed patients suffering from stoke, etc by helping them to lift the objects in the vicinity of the patient by using the bio-potentials ,thereby controlling the movement of the designed robot. The data acquired are analyzed in real time using a FPGAbased platform. By exploiting the qualities of MULTISIM functionality, design complexity, the design time of these signal circuits can be significantly reduced. Also a comparative analysis is done to prove the design of the hardware developed is accurate to an extent of $90 \%$. The paper aims at the verification and analysis of the developed/designed smart sensors with the design employed using MULTISIM package developed by NI-lab VIEW.
\end{abstract}

Keywords: Patient care and monitoring, Bed side monitors, Central nurse station, Zigbee, Electrooculogram.

\section{INTRODUCTION}

The availability of prompt and expert medical care can meaningfully improve health care services particularly at understaffed rural or remote areas. Continents facing continuous threats due to spread of infectious diseases, high levels of infant and maternal mortality, low level of life expectancy and deteriorating health care facilities are the greatest beneficiaries of continuous patient care monitoring and assisted with quick diagnosis techniques whenever required. To handle emergency situation, the main requirement is to continuously monitor intensive care parameters of patients and simultaneously display all captured information to the competent or decision making experts (doctors) at anywhere any time. A closer look at the patient monitoring system revels that it is the doctor alone who is remotely located whereas the emergency site is at a stable point. Recent advances in embedded computing systems have led to the emergence of wireless sensor networks consisting of small, battery powered "notes" with limited computation and radio communication capabilities. Sensor networks permits data gathering and computation to be deeply embedded in the physical environment. This technology has the potential to impact the delivery and study of resuscitative care by allowing vital signs to be automatically connected and fully integrated into the patient care record and used for real time storage, correlation of hospital records and long term observation.

This work is motivated by the following profound needs of patient monitoring systems namely:

i) The number of patients being managed at home is increasing in an effort to cut part the high hospitalization cost without compromising patients comfort. ii) Device non invasive waves to measure the different critical parameters and

iv) To design wearable vital sign sensors that can collect and relay over a short range wireless network to any number of receiving, including PDA'S, laptops, or ambulance-based terminals.

v) To give the capability for the data to be displayed in real time and also integrated into the developing pre-hospital patient care record.

vi)To give capability for the sensor devices themselves to be programmable, to process the vital sign data, for example, to raise an alert condition when vital signs fall outside of normal parameters.

vii) To device hardware architectures with ultra-low-power sensing, computation, and communication capability.

Patient care and monitoring has become an useful area of research to provide the patients a comfortable environment. The advent of embedded system's has made way to the innovation of FPGA's which can cater to a number of patients in an hospital environment specially in central nurse stations (CNS). Much research is under way into means of enabling the disabled to communicate effectively with a computer [1], as development of such means has the potential to enhance their quality of life considerably.

Patient care for paralyzed patients is a must for paralytic users with sufficient control of their eye movements; one option is to employ electrooculography (EOG) which uses eye movement measurements. It is usually done with a pair of electrodes that are placed either above and below the eye or to the left and right of the eye[2]. If the eye is moved from the center position towards one electrode, this electrode "sees" the positive side of the retina and the opposite electrode "sees" the negative side of the retina. Consequently, a potential difference occurs between the electrodes. Assuming that the resting potential is constant, the recorded potential is a measure for the eye position. EOG is a widely and successfully implemented technique and has proven reliable and easy to use in humanmachine interfaces (HMI) [3-5].

\section{METHODOLOGY}

\subsection{Data acquisition}

The purpose of this research paper is to develop a system to capture and analyze EOG signals in order to implement a HMI.Figure1 depicts the entire architecture of HMI. The system comprises two modules i.e. the signal Acquisition Module and the signal processing Module. Eyewear incorporating a set of appropriately positioned dry electrodes captures the EOG signals, which the acquisition module acquires, digitizes and transmits using the ZigBee protocol. 
The system will get input from the electronic signals produced by moving eyes and the system will act according to the signals which help the subject to operate assistive/interfaced devices. Modern eye tracking uses contrast to locate the centre of the pupil and uses near IR non collimated light to create a corneal reflection. Figure 2 shows the transmitter and the driver circuit for EOG data acquisition. A transmitter which generates oscillations of $2 \mathrm{kHz}$ is as shown in Fig 2.The transmitter sends infrared signal in a cone with a $25^{\circ}$ beam angle. The receiver reacts to infrared signals in a horizontal range of $\pm 30^{\circ}$ and in a vertical range of $\pm 35^{\circ}$. To ensures that the infrared signals can be reliably detected, the distance between transmitter and receiver can be varied between 0 and $3.5 \mathrm{~m}$. IR detector will give an analog output. Then, the analog output will through to the comparator to convert a digital output. Led will be on if get ' 1 ' and will be off if get ' 0 ' digital value.

The receiver circuit for EOG data acquisition is as shown in Fig 3.The positive input is given to IN4007 rectifier which gives positive peaks to the voltage regulator.The IR sensor senses the signals and these signals are amplified at the amplifier stage. The combined signals are fed to diode and peak rectifier. The ripple at the output of the rectifier is compared at the comparator.The comparator output is taken as the switching output which can be fed to a relay.

Fig4 shows the designed EEG system.EEG is recorded by mounting a sensor on the scalp electrodes . The frequency range of these brain waves is from $0-30 \mathrm{~Hz}[6-8]$. The electrode skin impedance usually is of the range $1 \mathrm{~K}$ to $10 \mathrm{~K}$.EEG amplifiers must have high input impedance typically about $10 \mathrm{M}$, so that the signal is not degraded. The electrodes are used as signal transducers which convert ionic currents into electronic current. The placement of the electrode is on the scalp of the patient and the ground electrode is placed at the mastoid behind the ears to avoid interference/artifacts

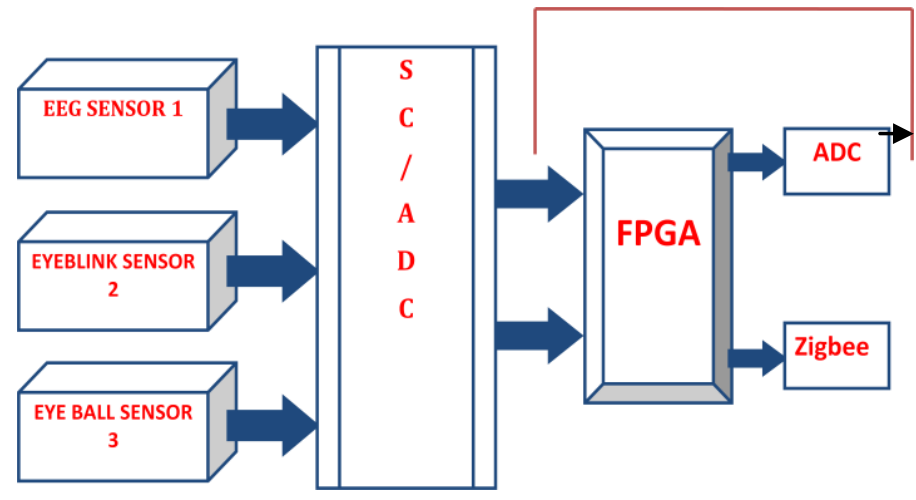

Figure 1. System architecture

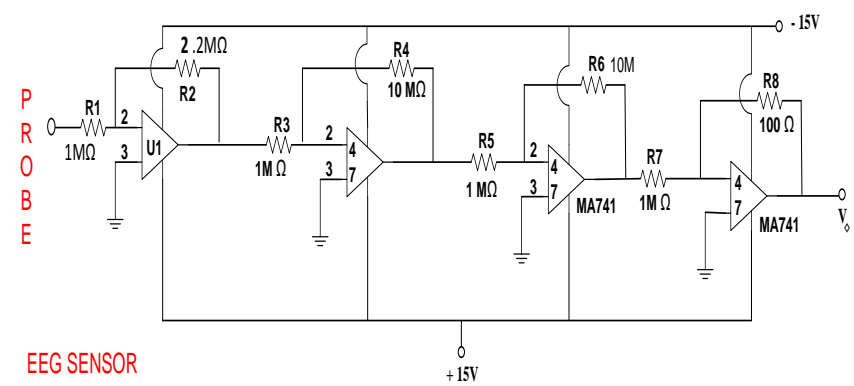

Figure 2. Designed EEG Sensor.

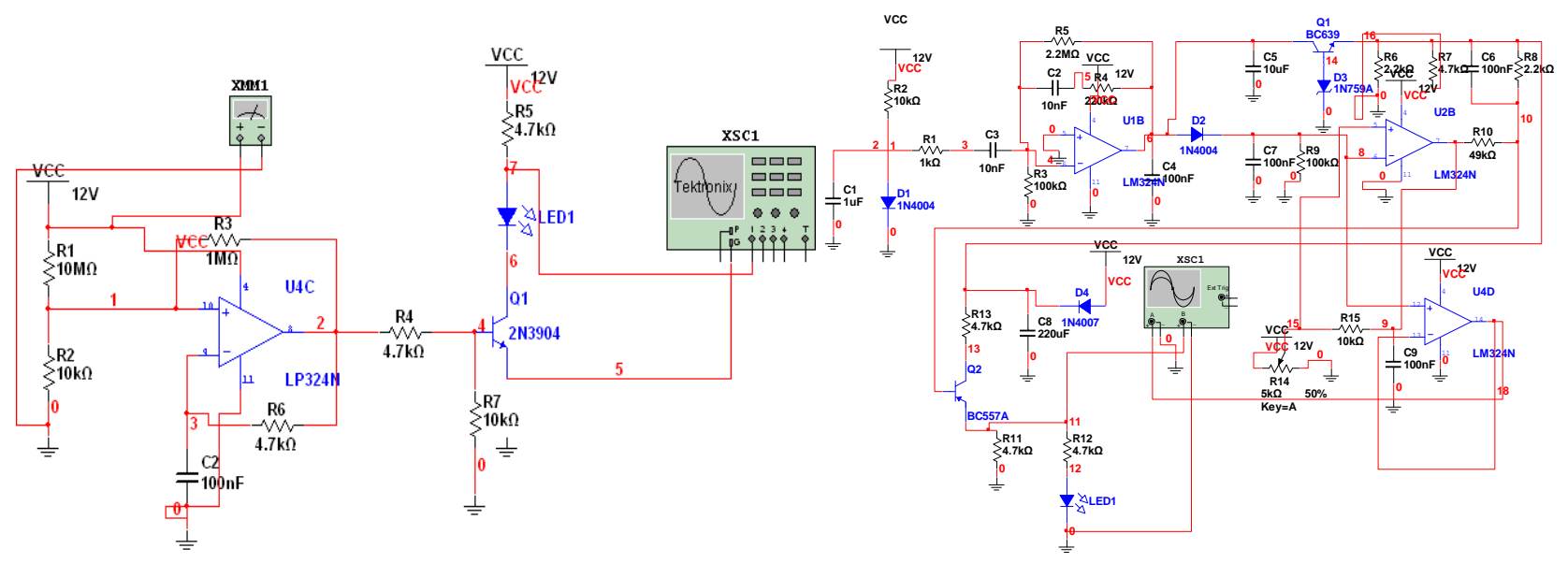

Figures 3 and 4: Transmitter and Receiver of EOG Fabricated Sensor design using MULTISIM 


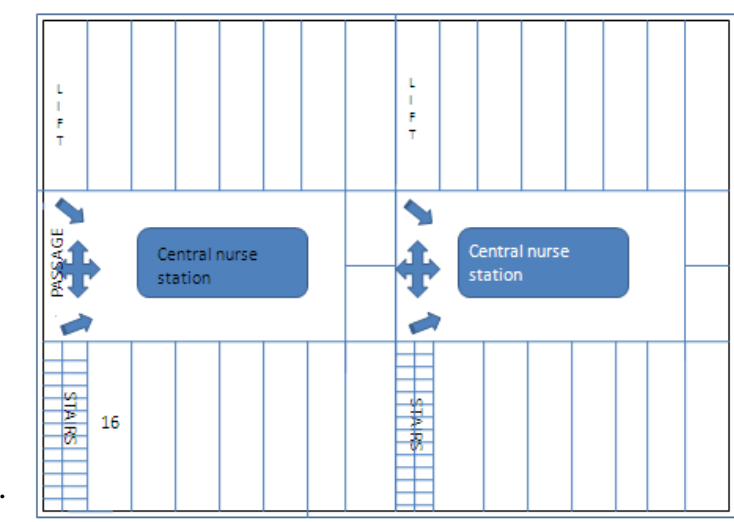

Figures 5: Architecture of dedicated hospital floor for paralytic patients.

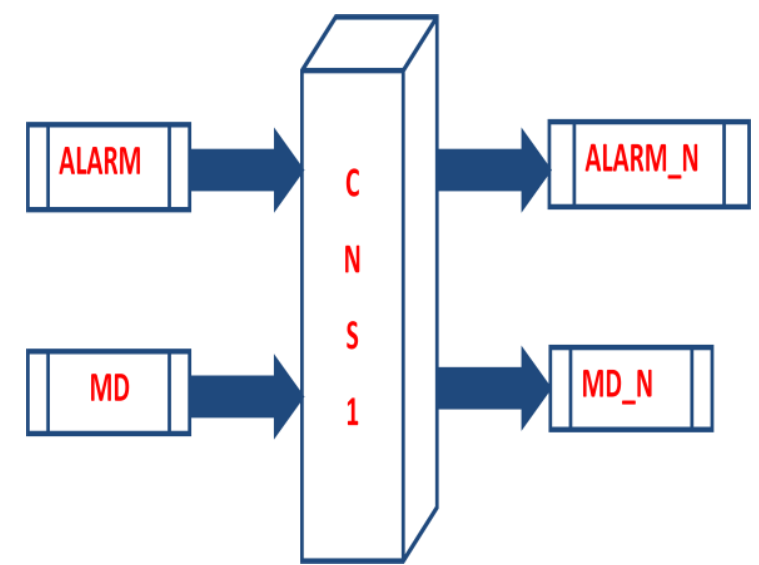

Figure 6: Main Display in CNS
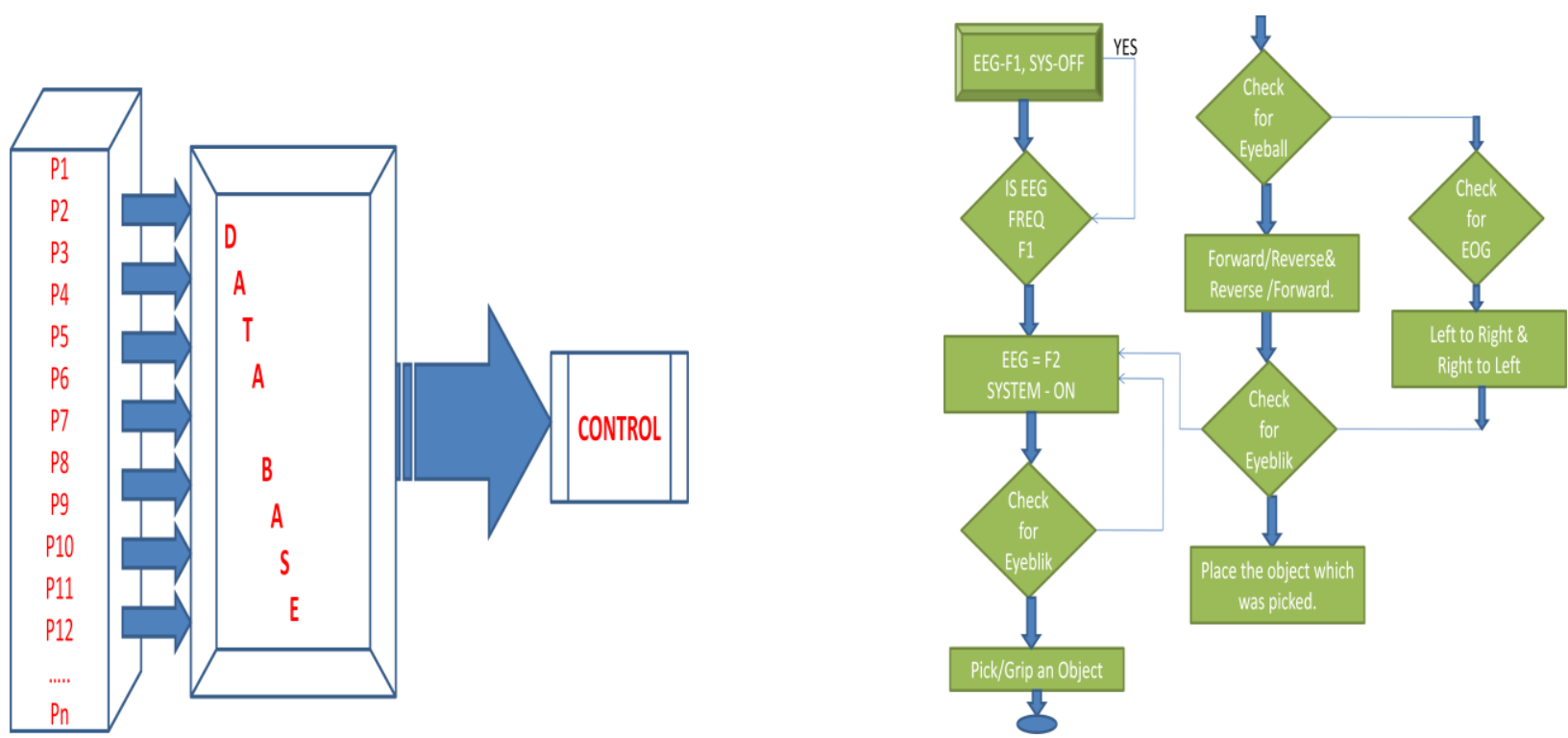

Figure 7 : Architecture of Central nurse station with Main display.

Figure 8: Algorithm for patient care system

\section{RESULTS}

\subsection{Assumptions done in design}

- Architectural design of the hospital done in such a way that a particular floor is being assigned to partially paralytic patients only.

- Microprocessor based robotic assistance controlled by various bio-potentials will be provided in each individual room of the hospital which can cater to on PPP.

- The entire floor operations i.e. from each room, such as Diagnosis, monitoring, Control and therapy (if possible) will be done using FPGA. FPGA is programmed to display any cases of emergency, such as alarm from a particular room, voice annunciation etc

For patient monitoring, the architecture of a complete floor dedicated for paralytic patients is as shown in fig5.This architecture helps in providing robotic assistance to individual patients in the room of a hospital. An architecture for main display (MD) is depicted in fig 6.The database to be updated with the records of the patient is controlled using FPGA.The algorithm in fig 8 shows the entire the entire flow of biopotentials and its working.

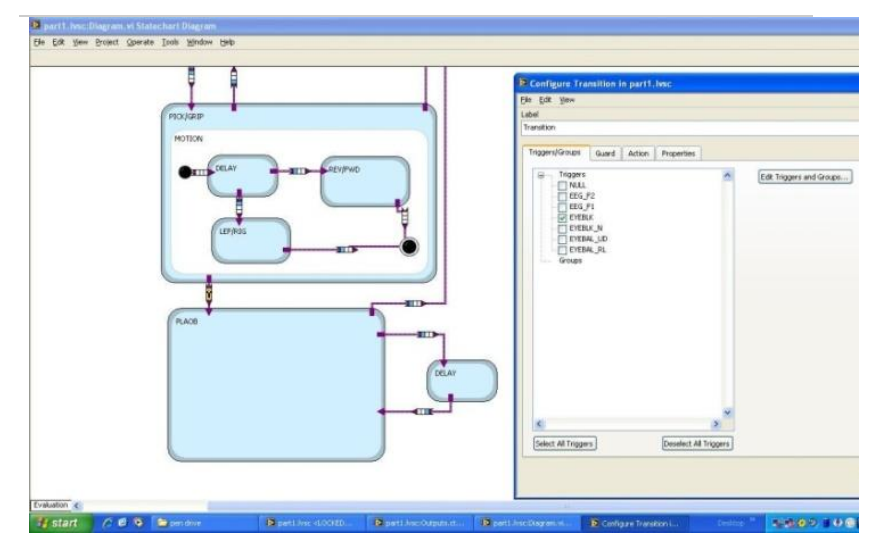

Fig.9 Ocular Artifact Removal Methods 
The elegant method of removal is to have trained technicians manually detect and remove epochs of corrupted data based on artifact characteristics such as amplitude, signal variance, frequency content, or slope that exceed a certain threshold [910].This extremely arduous and very subjective task leads to a significant amount of data loss, especially when there is a limited amount of data or a high frequency of blinking and saccades .The artifact removal is done using appropriate filters in the sensor design[11-15].

The analysis of the circuit of fig $2 \& 3$ implemented in MULTISIM is as in fig 10.The signal processing at each stage was checked and waveforms plotted at each output on the front panel is as shown in fig 9

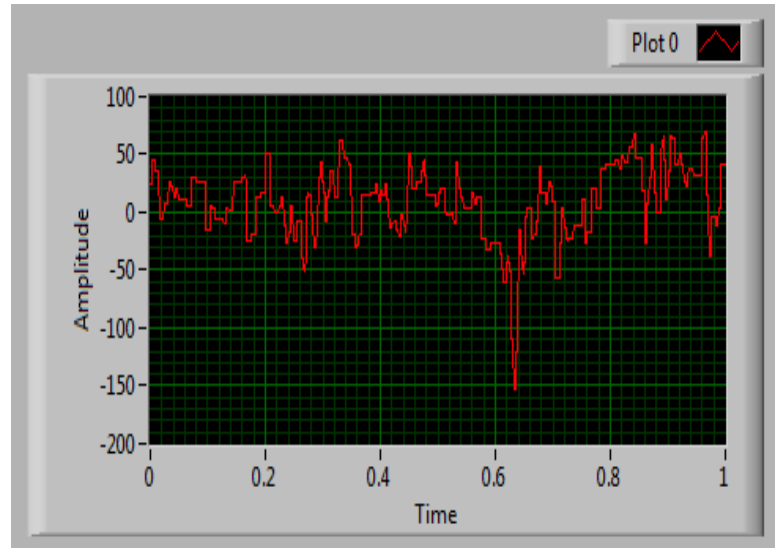

Fig 10: Front panel of EEG healthy patient eye open, EEG in upper waveform graph and lower waveform graph represent removal of artifact from EEG data. EEG data in upper waveform graph does not immediately convey useful information.

\section{EEG OF HEALTHY PATIENT, (EYES OPEN) USING INSTRUMENTATION VIRTUAL}

Fig 10 shows the EEG of a healthy patient. The EEG are contaminated by EOG signal. The EOG signal is a noncortical activity. The eye and brain activities have physiologically separate sources, so the EEG is a superposition of the true EEG and some portion of the EOG signal.

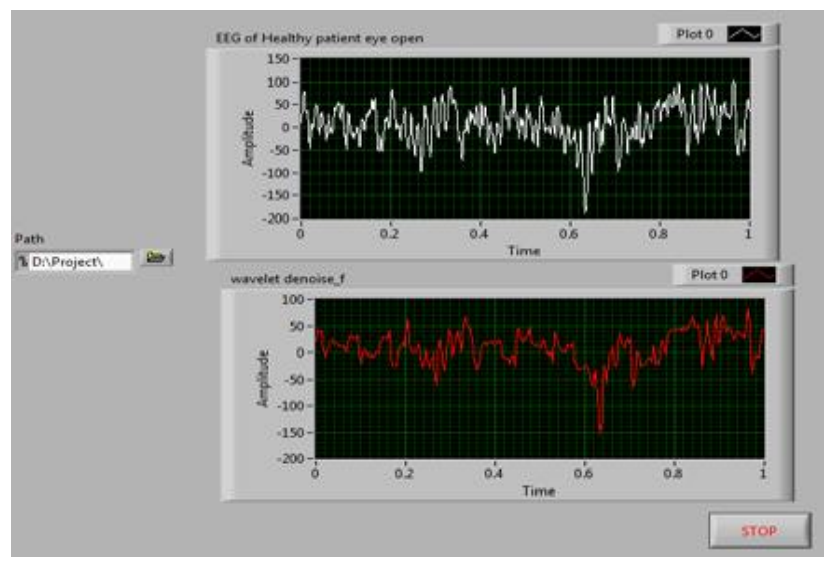

Fig 11: Front panel of EEG healthy patient eye open in lab VIEW using the fabricated sensor of fig $2 \& 3$
It can be represented as

EEGrec $(t)=$ EEGtrue $(t)+k . E O G(t) \quad--(1)$

Where, EEGrec(t) - contaminated EEG,

.EEGtrue(t)-EEG due to the cortical activity)

k.EOG (t) - Propagated ocular artifact from eye.

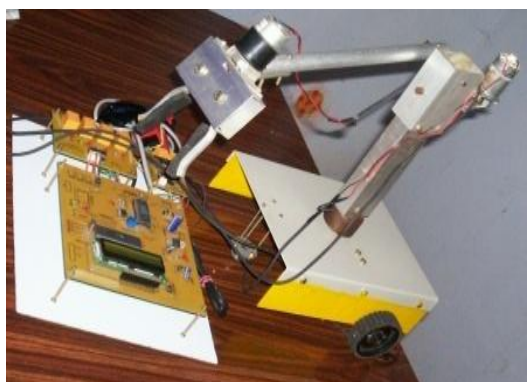

Fig 12: Fabricated sensor \&Robotic arm movement interfaced with microcontroller which moves with eye blink ,eyeball and EEG signals.

The system is powered $\mathrm{ON}$ if the patient is awake and is switched OFF if the patient is sleepy using the frequency output of EEG signals.

The eye blink helps in gripping and object thereby pick and place of an object is carried out. The eyeball movement from Left to right and from right to left is helping the gripper to move in accordance to the direction given by the eyeball of the patient.

\section{CONCLUSION AND FUTURE WORK}

The proposed work proved that the designed sensor worked with an accuracy of $98 \%$ and the system is reliable. The use of lab VIEW validated, the designed hardware which was fabricated using discrete components and the simulation results tallied with the real hardware. This gave the courage to the authors to implement the fabricated hardware on partially paralytic patients and test on them. Enhancement to this sensor is to implement a GUI(graphical user interface) and use of Image processing techniques to have an interactive VOD or games or audio on demand (juke box) and recreate a partially or a handicapped patient.

\section{REFERENCES}

[1] S. Venkataramanan, P. Prabhat, S. R. Choudhury, H. B. Nemade, and J. S. Sahambi, "Biomedical instrumentation based on Electrooculogram (EOG) signal processing and application to a hospital alarm system," in Proceedings of the 2nd IEEE International Conference on Intelligent Sensing and Information Processing (ICISIP '05), pp. 535-540, Chennai, India, January 2005.

[2] R. Barae, L. Boquete, and M. Mazo, "System for assisted mobility using eye movements based on electrooculography," IEEE Transaction on Neural Systems and Rehabilitation Engineering, vol. 10, no. 4, pp. 209-218, 2002.

[3] Y. Kim, N. L. Doh, Y. Youm, and W. K. Chung, "Robust discrimination method of the electrooculogram signals for human-computer interaction controlling mobile robot," Intelligent Automation and Soft Computing, vol. 13, no. 3, pp. 319-336, 2007.

[4] Z. Lv, X. Wu, M. Li, and C. Zhang, "Implementation of the EOG-based human computer interface system," in Proceedings of the 2nd International Conference on 
Bioinformatics and Biomedical Engineering (ICBBE '08), pp. 2188-2191, Shanghai, China, May 2008.

[5] C. K. Young and M. Sasaki, "Mobile robot control by neural network EOG gesture recognition," in Proceedings of the 8th International Conference on Neural Information Processing, vol. 1, pp. 322-328, 2001.

[6] Y. Chen and W. S. Newman, "A human-robot interface based on electrooculography," in Proceedings of the IEEE International Conference on Robotics and Automation (ICRA '04), pp. 243-248, New Orleans, La, USA, April 2004.

[7] D. Kumar and E. Poole, "Classification of EOG for human computer interface," in Proceedings of the 2nd Joint IEEE Annual International Conference of the Engineering in Medicine and Biology Jointly with the 24th Annual Conference of the Biomedical Engineering Society (BMES/EMBS '02), vol. 1, pp. 64-67, Houston, Tex, USA, October 2002.

[8] F. Cincotti, D. Mattia, F. Aloise, et al., "Non-invasive brain-computer interface system: towards its application as assistive technology," Brain Research Bulletin, vol. 75 , no. 6, pp. 796-803, 2008.

[9] A. B. Usakli and S. Gurkan, "Design of a novel efficient human computer interface: an electrooculagram based virtual keyboard," submitted to IEEE Transactions on Instrumentation and Measurement.

[10] F. De Vico Fallani, L. Astolfi, F. Cincotti, et al., "Cortical Functional Connectivity Networks In Normal

\section{ABOUT THE AUTHORS}

Mamatha. M. $\mathbf{N}$ received her M.E. degree in Electronics from University of Bangalore in 1999. She received her B.E. degree in Instrumentation from Mysore University in 1993. Presently, she is working as an assistant professor in B. M. S. College of engineering, Visveswaraya Technological University. She is presently doing a Ph. D. Research in Vinayaka Missions University, Salem, Tamilnadu. Her areas of interest are biomedical instrumentation and transducers. She has presented papers in International Conferences and journals.

Dr. S. Ramachandran has wide academic as well as industrial experience for over 30 years, having worked as Professor in various engineering colleges as well as design engineer in industries. Prior to this, he has been with the Indian Institute of Technology, Madras. He has industrial and teaching experience, having worked both in India and USA, designing systems and teaching/guiding students and practicing engineers based on FPGAs and Microprocessors. His research interests include developing algorithms, architectures and implementations on FPGAs/ASICs for Video Processing, DSP applications, reconfigurable computing, open loop control systems, etc. He has a number of papers in International Journals and Conferences. He is the recipient of the Best Design Award at VLSI Design 2000, International Conference held at Calcutta, India and the Best Paper Award of the Session at WMSCI 2006, Orlando, Florida, USA. He has completed a video course on Digital VLSI System Design at the Indian Institute of Technology Madras, India for broadcast on TV by National Programme on Technology on Enhanced Learning (NPTEL). He has also written a book on Digital VLSI Systems Design,
And Spinal Cord Injured Patients: Evaluation by Graph Analysis," Human Brain Mapping, vol. 28, no. 12, pp. 1334-1346, 2007.

[11] L. Astolfi, F. De Vico Fallani, F. Cincotti, et al., "Imaging Functional Brain Connectivity Patterns From High-Resolution EEG And fMRI Via Graph Theory," Psychophysology, vol. 44, no. 6, pp. 880-893, 2007.

[12] L. Astolfi, F. Cincotti, D. Mattia, et al., "Tracking the time-varying cortical connectivity patterns by adaptive multivariate estimators," IEEE Trans on Biomedical Engineering, vol. 55, no. 3, pp. 902-913, 2008.

[13] M. Oliveri, C. Babiloni, M. M. Filippi, et al., "Influence of the supplementary motor area on primary motor cortex excitability during movements triggered by neutral or emotionally unpleasant visual cues," Exp Brain Res, vol. 149 , no. 2, pp. 214-221, 2003.

[14] C. Babiloni, F. Babiloni, F. Carducci, et al., "Mapping of early and late human somatosensory evoked brain potentials to phasic galvanic painful stimulation," Human Brain Mapping, vol. 12, no. 3, pp. 168-179, 2001.

[15] A. Urbano, C. Babiloni, F. Carducci, L. Fattorini, P. Onorati, and F. Babiloni, "Dynamic functional coupling of high resolution EEG potentials related to unilateral internally triggered one-digit movements," Electroencephalography and clinical Neurophysiol, vol. 106, pp. 477-487, 1998.

published by Springer Verlag, Netherlands (www.springer.com).

Dr M. Chandrasekaran received his B.E.(Hons.) degree in Electronics and communication engineering from University of Madras and his M.E. degree in Computer Science and Engineering from Bharathiar University. His area of research is in Information and Communication Engineering under Anna University. He has more than 20 years of teaching experience. He has published papers in National and International Conferences conducted by IEEE. He is a member of ISTE and CSI. His research interests include Neural networks, Fuzzy logic, Congestion control in TCP networks and Sensor networks. He served as member of various committees representing AICTE and DOTE, Tamil Nadu for inspecting Engineering colleges. He served as a member of Academic council. He guided many B.E., M.E. and M.C.A Projects. Some of the Projects got the Award from Tamil Nadu State Council for Science and Technology. He is currently working as Assosiate Professor in Electronics and communication Engineering department at Government College of Engineering, Salem 